\title{
LOWER BOUNDS ON THE MSE FOR MIXED FAR-FIELD AND NEAR-FIELD SOURCES DIRECTION-OF-ARRIVALS
}

\author{
Alexandre Renaux, Rémy Boyer and Sylvie Marcos \\ Université Paris-Sud / CNRS / Supelec \\ Laboratory of Signals and Systems \\ 3, rue Joliot-Curie, 91 Gif-sur-Yvette, France
}

\begin{abstract}
Fundamental limits in terms of mean square error for source localization by an array of sensors are investigated. The context is the case of a far-field source and a near-field source for which few results are available. Particularly, in order to characterize the SNR threshold exhibited by estimators in such scenario, lower bounds other than the Cramér-Rao bound are studied.
\end{abstract}

\section{INTRODUCTION}

Very few works are related to the study of the realistic situation where there exists coexisting far-field (FF) and near-field (NF) sources such as speaker localization using microphone arrays and guidance (homing) systems. At the contrary, we can find a plethora of contributions on the localization of farfield sources [1][2]. One can note the existence of some estimation algorithms adapted to the passive near field source localization [3, 4, 5, 6, 7].

Nevertheless, there exist only few works studying the asymp totic estimation performance in this context $[5,8]$. In the context of the problem of source localization, one can see three contributions: (1) propose new efficient algorithms/estimators [9], (2) study the estimation performance independently of a specific algorithm thanks to the lower bound on the mean square error [10][11][12][13][14] and (3) derive and study the theoretical resolution, i.e., the minimal angular distance to resolve/discriminate two closely spaced emitted signals in terms of their direction of arrivals [15][16]. Our contribution belongs to the second point.

More precisely, to characterize the asymptotic performance of an estimator in terms of the mean square error, the CramérRao bound [17], which is asymptotically achieved under certain mild/general conditions [18][19], is the most popular tool. However, the Cramér-Rao bound becomes too optimistic in the non-asymptotic region (i.e., when the outlier effect appears.) This non-asymptotic region is delimited by the socalled threshold or breakdown point (i.e., when the estima-

This project is funded by both the Digiteo Research Park and the Region Ile-de-France. tor's mean square error increases dramatically [20].) One should note that the prediction of this threshold is of great important since it delimits the optimal operating zone of the estimators. We consider the two classical source signal model assumptions [21]: the conditional model (i.e., when the signals are assumed to be deterministic) and the unconditional model (i.e., when the signals are assumed to be driven by a Gaussian random process). For each model, we propose to characterize the threshold region using deterministic lower bounds on the estimator's mean square error. In particular, we derive and analyze the Barankin bound [22][23][24] which is known to be tighter than the Cramér-Rao bound and which exhibit the threshold effect.

\section{PROBLEM SETUP}

One considers the scenario where several signal sources, denoted $s_{F F_{k}}(t)$ and $s_{N F_{l}}(t)$, with $k=1, \ldots, K$ and $l=$ $1, \ldots, L$ are received by an uniform linear array made from $J$ identical and omnidirectional sensors with inter-element spacing denoted $d$. The source signals are assumed to be narrow-bands and $T$ snapshots are available. $K$ sources are located in the far-field area while the $L$ others are located in the near-field area. Consequently, we are interested by all the direction of arrivals of each sources and by the ranges of the sources located in the near-field area. Concerning the sources located in the far-field area, the noise-free observations for one snapshot is given by $\sum_{k=1}^{K} \mathbf{a}\left(\omega_{F F_{k}}\right) s_{F F_{k}}(t)$ where $\mathbf{a}\left(\omega_{F F_{k}}\right)$ is the steering vector $(J \times 1)$ with the $j^{\text {th }}$ element given by $\left\{\mathbf{a}\left(\omega_{F F_{k}}\right)\right\}_{j}=\exp \left(i \omega_{F F_{k}} j\right)$ for $j=0, \ldots, J-1$. The so-called electric parameters $\omega_{F F_{k}}$ can be linked to the direction of arrival, $\theta_{F F_{k}}$, of the $k^{t h}$ far-field source according to $\omega_{F F_{k}}=-\frac{2 \pi d}{\lambda} \sin \left(\theta_{F F_{k}}\right)$. Concerning the sources located in the near-field area, the noise-free observations for one snapshot is given by $\sum_{l=1}^{L} \mathbf{b}\left(\omega_{N F_{l}}, \phi_{N F_{l}}\right) s_{N F_{l}}(t)$ where $\mathbf{b}\left(\omega_{N F_{l}}, \phi_{N F_{l}}\right)$ is the steering vector $(J \times 1)$ with the $j^{t h}$ element given by

$$
\left\{\mathbf{b}\left(\omega_{N F_{l}}, \phi_{N F_{l}}\right)\right\}_{j}=\exp \left(i\left(\omega_{N F_{l}} j+\phi_{N F_{l}} j^{2}\right)\right)
$$


for $j=0, \ldots, J-1$. Again, note that the so-called electric parameters $\omega_{l}$ and $\phi_{l}$ can be linked to both the direction of arrival, $\theta_{N F_{l}}$, and the range, $r_{N F_{l}}$, of the near-field source according to $\omega_{N F_{l}}=-\frac{2 \pi d}{\lambda} \sin \left(\theta_{N F_{l}}\right)$ and $\phi_{N F_{l}}=$ $\frac{\pi d^{2}}{\lambda r_{N F_{l}}} \cos ^{2}\left(\theta_{N F_{l}}\right)$. Note that, while the structure of $\mathbf{a}\left(\omega_{F F_{k}}\right)$ is well known in array processing, the structure of the steering vector $\mathbf{b}\left(\omega_{N F_{l}}, \phi_{N F_{l}}\right)$ is obtained by assuming, like most of the algorithms in the literature, that the ranges lies on the Fresnel region, i.e., $0.62\left(d^{3}(L-1)^{3} / \lambda\right)^{1 / 2}<r_{N F_{l}}<2 d^{2}(L-$ $1)^{2} / \lambda \forall l$. Finally, the observation model for our scenario is the following

$$
\begin{aligned}
\mathbf{y}(t)= & \sum_{k=1}^{K} \mathbf{a}\left(\omega_{F F_{k}}\right) s_{F F_{k}}(t) \\
& +\sum_{l=1}^{L} \mathbf{b}\left(\omega_{N F_{l}}, \phi_{N F_{l}}\right) s_{N F_{l}}(t)+\mathbf{n}(t)
\end{aligned}
$$

$t=1, \ldots, T$, where $\mathbf{y}(t)$ is the observed antenna signal $(J \times 1)$ for one snapshot and where $\mathbf{n}(t)$ is the additive noise $(J \times 1)$. Our assumptions are the following: (i) the signal sources, $s_{F F_{k}}(t)$ and $s_{N F_{l}}(t)$, are assumed to be temporally white complex Gaussian circular, jointly uncorrelated, with zero mean and known variances $\sigma_{F F_{k}}^{2}$ and $\sigma_{N F_{l}}^{2} \forall k$ and $\forall l,(i$ bis) the signal sources, $s_{F F_{k}}(t)$ and $s_{N F_{l}}(t)$, are assumed to be deterministic and known $\forall t, \forall k$ and $\forall l$ (ii) the noise, $\mathbf{n}(t)$, is assumed temporally white complex circular Gaussian (uncorrelated with respect to the source signals) with zero mean and (known) covariance matrix $\sigma^{2} \mathbf{I}_{J \times J}$. The $K+2 L$ parameters of interest are $\left\{\omega_{F F_{k}}\right\}_{k=1, \ldots, K},\left\{\omega_{N F_{l}}\right\}_{l=1, \ldots, L}$ and $\left\{\phi_{N F_{l}}\right\}_{l=1, \ldots, L}$. They are assumed to be deterministic.

\section{BARANKIN BOUND}

\subsection{Background}

In the non-Bayesian context (deterministic unknown parameters) the Barankin bound is the greatest lower bound for any globally unbiased estimator. In this paper, we will use the so-called McAulay-Seidman bound [23] which is a useful approximation of the Barankin bound. Let $\hat{\boldsymbol{\theta}}$ be an unbiased estimator of a vector of interest $\boldsymbol{\theta}$ of size $P \times 1$, then one has the following inequality about the estimation error covariance matrix

$$
\mathbb{E}\left[(\hat{\boldsymbol{\theta}}-\boldsymbol{\theta})(\hat{\boldsymbol{\theta}}-\boldsymbol{\theta})^{T}\right] \succeq \mathbf{H} \boldsymbol{\Phi}^{-1} \mathbf{H}^{T}
$$

where $\mathbf{A} \succeq \mathbf{B}$ means that the matrix $\mathbf{A}-\mathbf{B}$ is non-negative defined. In the right hand side of Eqn. (2), $\mathbf{H}$ is a $P \times Q$ matrix containing the so-called $Q$ test-points left to the user. This matrix will be structured has follows:

$$
\mathbf{H}=\left(\begin{array}{llll}
\mathbf{h}_{1} & \mathbf{h}_{2} & \cdots & \mathbf{h}_{Q}
\end{array}\right) .
$$

Generally, these test points are choosed uniformly distributed over the parameter space $\Theta$ such that $\boldsymbol{\theta}+\mathbf{h}_{q} \in \Theta$, $\forall q=1, \ldots, Q$. Moreover higher is the number of test points, tighter will be the bound to predict the threshold effect. On the other hand, $\Phi$ is a $Q \times Q$ matrix that one has to inverse, so if $Q$ is high, the computation of the bound becomes computationally expensive. The elements of $\Phi$ are given by

$$
\{\Phi\}_{m, n}=\int_{\Omega} \frac{p\left(\mathbf{Y} ; \boldsymbol{\theta}+\mathbf{h}_{m}\right) p\left(\mathbf{Y} ; \boldsymbol{\theta}+\mathbf{h}_{n}\right)}{p(\mathbf{Y} ; \boldsymbol{\theta})} d \mathbf{Y}-1,
$$

where $\mathbf{Y}=\left(\begin{array}{lll}\mathbf{y}(1) & \cdots & \mathbf{y}(T)\end{array}\right), p(\mathbf{Y} ;$.$) is the joint prob-$ ability density function of the full set of observations and $\Omega$ is the observation space.

\subsection{Application to our problem}

In the context of the aforementioned scenario, we have

$$
\boldsymbol{\theta}=\left(\omega_{F F_{k}} \cdots \omega_{F F_{k}} \omega_{N F_{l}} \cdots \omega_{N F_{l}} \phi_{N F_{l}} \cdots \phi_{N F_{l}}\right)^{T},
$$

(and consequently, $P=K+2 L$ ).

First, under the assumption (i), i.e. that the signal sources, $s_{F F_{k}}(t)$ and $s_{N F_{l}}(t)$, are assumed to be temporally white complex Gaussian circular, jointly uncorrelated, with zero mean and known variances $\sigma_{F F_{k}}^{2}$ and $\sigma_{N F_{l}}^{2} \forall k$ and $\forall l$, and due to the noise assumptions, it is clear that the joint probability density function of the full set of observations is the product of the probability density functions of one observation vector over $t=1, \ldots, T$ and that the probability density function of one observation vector (i.e. for one snapshot $\mathbf{y}(t)$ ) is complex Gaussian circular with zero mean and covariance matrix $\boldsymbol{\Sigma}_{\mathbf{y}}(\boldsymbol{\theta})$ given by

$$
\begin{gathered}
\boldsymbol{\Sigma}_{\mathbf{y}}(\boldsymbol{\theta})=\sum_{k=1}^{K} \sigma_{F F_{k}}^{2} \mathbf{a}\left(\omega_{F F_{k}}\right) \mathbf{a}^{H}\left(\omega_{F F_{k}}\right) \\
+\sum_{l=1}^{L} \mathbf{b}\left(\omega_{N F_{l}}, \phi_{N F_{l}}\right) \mathbf{b}^{H}\left(\omega_{N F_{l}}, \phi_{N F_{l}}\right)+\sigma^{2} \mathbf{I}_{J \times J} .
\end{gathered}
$$

In other words,

$$
\begin{gathered}
\{\Phi\}_{m, n}= \\
\prod_{t=1}^{T} \int_{\Omega} \frac{p\left(\mathbf{y}(t) ; \boldsymbol{\theta}+\mathbf{h}_{m}\right) p\left(\mathbf{y}(t) ; \boldsymbol{\theta}+\mathbf{h}_{n}\right)}{p(\mathbf{y}(t) ; \boldsymbol{\theta})} d \mathbf{y}(t)-1,
\end{gathered}
$$

and

$$
p(\mathbf{y}(t) ; \boldsymbol{\theta})=\frac{1}{\pi^{J}\left|\boldsymbol{\Sigma}_{\mathbf{y}}(\boldsymbol{\theta})\right|} \exp \left(-\mathbf{y}^{H}(t) \boldsymbol{\Sigma}_{\mathbf{y}}^{-1}(\boldsymbol{\theta}) \mathbf{y}(t)\right)
$$

where $\boldsymbol{\Sigma}_{\mathbf{y}}(\boldsymbol{\theta})$ is given by Eqn. (5). It is easy to see that

$$
\begin{gathered}
\int_{\Omega} \frac{p\left(\mathbf{y}(t) ; \boldsymbol{\theta}+\mathbf{h}_{m}\right) p\left(\mathbf{y}(t) ; \boldsymbol{\theta}+\mathbf{h}_{n}\right)}{p(\mathbf{y}(t) ; \boldsymbol{\theta})} d \mathbf{y}(t)= \\
\frac{\left|\boldsymbol{\Sigma}_{\mathbf{y}}(\boldsymbol{\theta})\right| \mid \boldsymbol{\Sigma}_{\mathbf{y}}\left(\boldsymbol{\theta}+\left.\mathbf{h}_{n}\right|^{-1}\right.}{\pi^{J}\left|\boldsymbol{\Sigma}_{\mathbf{y}}\left(\boldsymbol{\theta}+\mathbf{h}_{m}\right)\right|} \int_{\Omega} \exp \left(-\mathbf{y}^{H}(t) \boldsymbol{\Gamma}^{-1} \mathbf{y}(t)\right) d \mathbf{y}(t),(8)
\end{gathered}
$$


where $\boldsymbol{\Gamma}^{-1}=\boldsymbol{\Sigma}_{\mathbf{y}}^{-1}\left(\boldsymbol{\theta}+\mathbf{h}_{m}\right)+\boldsymbol{\Sigma}_{\mathbf{y}}^{-1}\left(\boldsymbol{\theta}+\mathbf{h}_{n}\right)-\boldsymbol{\Sigma}_{\mathbf{y}}^{-1}(\boldsymbol{\theta})$. Then, since

$$
\int_{\Omega} \exp \left\{-\mathbf{y}^{H}(t) \boldsymbol{\Gamma}^{-1} \mathbf{y}(t)\right\} d \mathbf{y}(t)=\pi^{J}|\boldsymbol{\Gamma}|,
$$

one obtains the closed-form expression of $\{\Phi\}_{m, n}$ which is given by

$$
\{\Phi\}_{m, n}=\frac{\left|\boldsymbol{\Sigma}_{\mathbf{y}}(\boldsymbol{\theta})\right|^{T}\left|\boldsymbol{\Sigma}_{\mathbf{y}}\left(\boldsymbol{\theta}+\mathbf{h}_{m}\right)\right|^{-T}\left|\boldsymbol{\Sigma}_{\mathbf{y}}\left(\boldsymbol{\theta}+\mathbf{h}_{n}\right)\right|^{-T}}{\left|\boldsymbol{\Sigma}_{\mathbf{y}}^{-1}\left(\boldsymbol{\theta}+\mathbf{h}_{m}\right)+\boldsymbol{\Sigma}_{\mathbf{y}}^{-1}\left(\boldsymbol{\theta}+\mathbf{h}_{n}\right)-\boldsymbol{\Sigma}_{\mathbf{y}}^{-1}(\boldsymbol{\theta})\right|^{T}}+1 .
$$

Consequently, by plugging Eqn. (5) into to Eqn. (10), there are no more integrals to compute in order to obtain the bound.

Second, under the assumption (i-bis), i.e. that the signal sources, $s_{F F_{k}}(t)$ and $s_{N F_{l}}(t)$, are assumed to be deterministic and known $\forall t, \forall k$ and $\forall l$, and due to the noise assumptions, it is clear that the joint probability density function of the full set of observations is still the product of the probability density functions of one observation vector over $t=1, \ldots, T$ and that the probability density function of one observation vector (i.e. for one snapshot $\mathbf{y}(t)$ ) is complex Gaussian circular with mean $\boldsymbol{\eta}(t, \boldsymbol{\theta})$ given by

$$
\begin{gathered}
\boldsymbol{\eta}(t, \boldsymbol{\theta})= \\
\sum_{k=1}^{K} \mathbf{a}\left(\omega_{F F_{k}}\right) s_{F F_{k}}(t)+\sum_{l=1}^{L} \mathbf{b}\left(\omega_{N F_{l}}, \phi_{N F_{l}}\right) s_{N F_{l}}(t),
\end{gathered}
$$

and covariance matrix $\sigma^{2} \mathbf{I}_{J \times J}$. In other words, Eqn. (6) still holds and

$$
p(\mathbf{y}(t) ; \boldsymbol{\theta})=\frac{1}{\left(\pi \sigma^{2}\right)^{J}} \exp \left(-\frac{1}{\sigma^{2}}\|\mathbf{y}(t)-\boldsymbol{\eta}(t, \boldsymbol{\theta})\|^{2}\right) .
$$

After some easy calculus, one can see that

$$
\begin{aligned}
\sigma^{2} \ln \int_{\Omega} \frac{p(\mathbf{y}(t) ; \boldsymbol{\theta}+}{\left.\mathbf{h}_{m}\right) p\left(\mathbf{y}(t) ; \boldsymbol{\theta}+\mathbf{h}_{n}\right)} & p(\mathbf{y}(t) ; \boldsymbol{\theta}) \\
& \left\|\boldsymbol{\eta}\left(t, \boldsymbol{\theta}+\mathbf{h}_{m}\right)-\boldsymbol{\eta}(t, \boldsymbol{\theta})\right\|^{2} \\
& -\left\|\boldsymbol{\eta}\left(t, \boldsymbol{\theta}+\mathbf{h}_{m}\right)-\boldsymbol{\eta}\left(t, \boldsymbol{\theta}+\mathbf{h}_{n}\right)\right\|^{2} \\
& +\left\|\boldsymbol{\eta}\left(t, \boldsymbol{\theta}+\mathbf{h}_{n}\right)-\boldsymbol{\eta}(t, \boldsymbol{\theta})\right\|^{2} .
\end{aligned}
$$

Consequently,

$$
\begin{gathered}
\{\Phi\}_{m, n}= \\
\exp \frac{1}{\sigma^{2}} \sum_{t=1}^{T}\left(\begin{array}{c}
\left\|\boldsymbol{\eta}\left(t, \boldsymbol{\theta}+\mathbf{h}_{m}\right)-\boldsymbol{\eta}(t, \boldsymbol{\theta})\right\|^{2} \\
-\left\|\boldsymbol{\eta}\left(t, \boldsymbol{\theta}+\mathbf{h}_{m}\right)-\boldsymbol{\eta}\left(t, \boldsymbol{\theta}+\mathbf{h}_{n}\right)\right\|^{2} \\
+\left\|\boldsymbol{\eta}\left(t, \boldsymbol{\theta}+\mathbf{h}_{n}\right)-\boldsymbol{\eta}(t, \boldsymbol{\theta})\right\|^{2}
\end{array}\right) .
\end{gathered}
$$

Again, there are no more integrals to compute in order to obtain the bound.

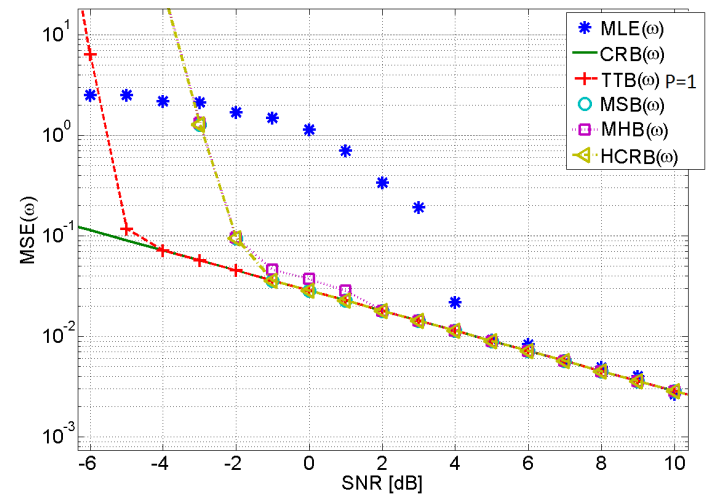

Fig. 1. Lower bounds on the mean square error (deterministic case) w.r.t. $\omega$ with 10 snapshots and $(\theta, r)=\left(45^{\circ}, 6 \lambda\right)$.

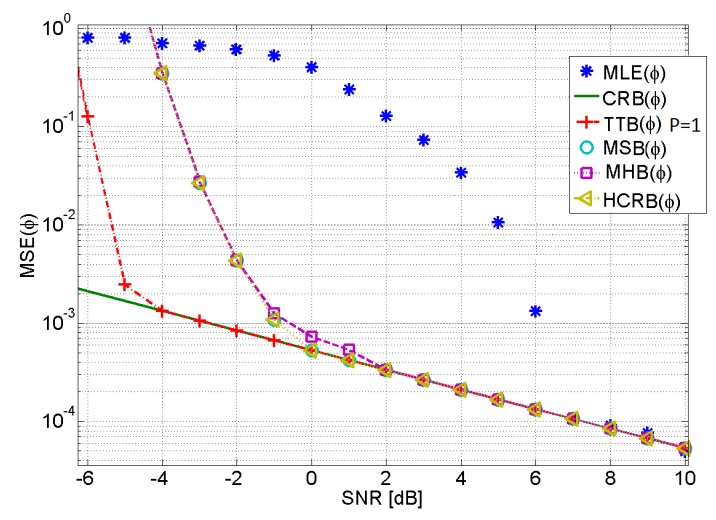

Fig. 2. Lower bounds on the mean square error (deterministic case) w.r.t. $\phi$, with 10 snapshots and $(\theta, r)=\left(45^{\circ}, 6 \lambda\right)$.

\section{SIMULATION RESULTS}

The scenario used in these simulations is a uniform linear array of 5 sensors spaced by $\frac{\lambda}{2}$.

To compare the threshold prediction accuracy we plot the MSE w.r.t. $\omega$ and $\phi$ using 1000 Monte Carlo trials. Both deterministic and stochastic cases are considered. We compare our bound [23] to several well known bounds in the literature computed numerically (particularly [25] and [26]).

\section{CONCLUSION}

In this paper, one gives closed-form expressions of the Barankin bound in the context of a scenario where there exists coexisting far-field and near-field sources. Both the stochastic and deterministic observations model cases are studied and we compare the bound with several other of the literature. 


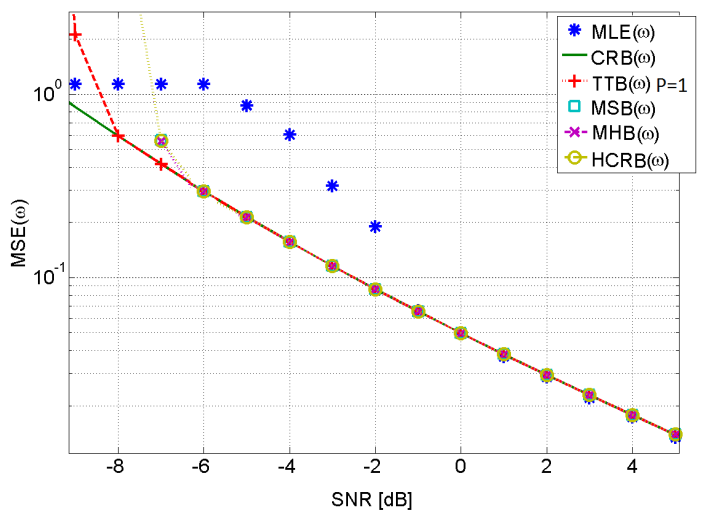

Fig. 3. Lower bounds on the mean square error (the stochastic case) w.r.t. $\omega$ with 100 snapshots and $(\theta, r)=\left(30^{\circ}, 6 \lambda\right)$.

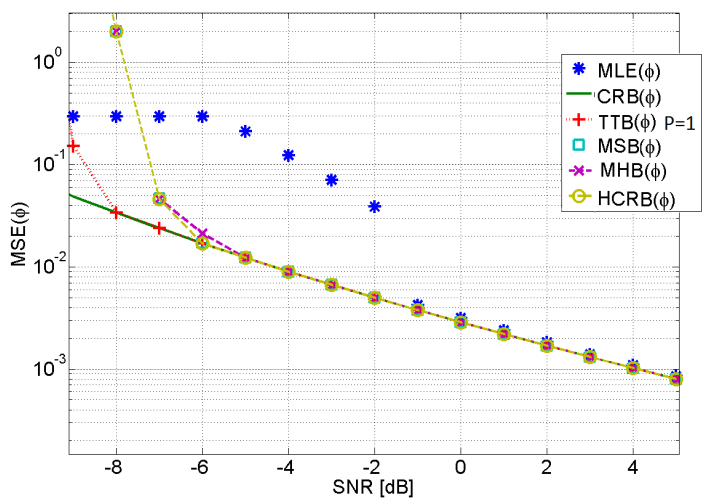

Fig. 4. Lower bounds on the mean square error (the stochastic case) w.r.t. $\phi$ with $T=100$ snapshots and $(\theta, r)=\left(30^{\circ}, 6 \lambda\right)$.

\section{REFERENCES}

[1] P. Stoica and A. Nehorai, "MUSIC, maximum likelihood and the Cramér-Rao bound," IEEE Transactions on Acoustics, Speech, and Signal Processing, vol. 37, pp. 720-741, May 1989.

[2] H. L. Van Trees, Detection, Estimation and Modulation theory: Optimum Array Processing, vol. 4. NewYork, NY, USA: John Wiley \& Sons, Mar. 2002.

[3] Y. D. Huang and M. Barkat, "Near-field multiple source localization by passive sensor array," vol. 39, pp. 968975, 1991.

[4] N. Yuen and B. Friedlander, "Performance analysis of higher order ESPRIT for localization of near-field sources," vol. 46, pp. 709-719, 1998.

[5] E. Grosicki, K. Abed-Meraim, and Y. Hua, "A weighted linear prediction method for near-field source localization," vol. 53, pp. 3651-3660, 2005.

[6] W. Zhi and M. Chia, "Near-field source localization via symmetric subarrays," vol. 14, no. 6, pp. 409-412, 2007.

[7] M. N. El Korso, G. Bouleux, R. Boyer, and S. Marcos, "Sequential estimation of the range and the bearing using the zero-forcing MUSIC approach," in Proc. EUSIPCO, (Glasgow, Scotland), pp. 1404-1408, Aug. 2009.

[8] M. N. El Korso, R. Boyer, A. Renaux, and S. Marcos, "Nonmatrix closed-form expressions of the CramérRao bounds for near-field localization parameters," in Proc. of IEEE International Conference on Acoustics, Speech, and Signal Processing (ICASSP), (Taipei, Taiwan), 2009.

[9] H. Krim and M. Viberg, "Two decades of array signal processing research: the parametric approach," IEEE Signal Processing Magazine, vol. 13, pp. 67-94, July 1996.

[10] H. L. Van Trees and K. L. Bell, eds., Bayesian Bounds for Parameter Estimation and Nonlinear Filtering/Tracking. New-York, NY, USA: Wiley/IEEE Press, Sept. 2007.

[11] J. Tabrikian and J. L. Krolik, "Barankin bounds for source localization in an uncertain ocean environment," IEEE Transactions on Signal Processing, vol. 47, pp. 2917-2927, Nov. 1999.

[12] D. T. Vu, A. Renaux, R. Boyer, and S. Marcos, "Performance analysis of $2 \mathrm{D}$ and $3 \mathrm{D}$ antenna arrays for source localization," in Proc. of European Signal Processing Conference (EUSIPCO), (Aalborg, Denmark), Sept. 2010. 
[13] A. Renaux, P. Forster, and P. Larzabal, "A new derivation of the Bayesian bounds for parameter estimation," in Proc. of IEEE Workshop on Statistical Signal Processing (SSP), (Bordeaux, FR), pp. 567-572, July 2005.

[14] A. Renaux, P. Forster, P. Larzabal, and C. D. Richmond, "The Bayesian Abel bound on the mean square error," in Proc. of IEEE International Conference on Acoustics, Speech, and Signal Processing (ICASSP), vol. 3, (Toulouse, FR), pp. 9-12, May 2006.

[15] S. T. Smith, "Statistical resolution limits and the complexified Cramér-Rao bound," IEEE Transactions on Signal Processing, vol. 53, pp. 1597-1609, May 2005.

[16] M. N. El Korso, R. Boyer, A. Renaux, and S. Marcos, "Statistical resilution limit for multiple signals and parameters of interest," in Proc. of IEEE International Conference on Acoustics, Speech, and Signal Processing (ICASSP), (Dallas, TX), 2010.

[17] H. Cramér, Mathematical Methods of Statistics, vol. 9 of Princeton Mathematics. New-York: Princeton University Press, Sept. 1946.

[18] E. L. Lehmann and G. Casella, Theory of Point Estimation. Springer Texts in Statistics, New-York, NY, USA: Springer, 2 ed., Sept. 2003.

[19] A. Renaux, P. Forster, E. Chaumette, and P. Larzabal, "On the high-SNR conditional maximum-likelihood estimator full statistical characterization," IEEE Transactions on Signal Processing, vol. 54, pp. 4840-4843, Dec. 2006.

[20] D. C. Rife and R. R. Boorstyn, "Single tone parameter estimation from discrete time observations," IEEE Transactions on Information Theory, vol. 20, pp. 591598, Sept. 1974.

[21] B. Ottersten, M. Viberg, P. Stoica, and A. Nehorai, "Exact and large sample maximum likelihood techniques for parameter estimation and detection in array processing," in Radar Array Processing (S. S. Haykin, J. Litva, and T. J. Shepherd, eds.), ch. 4, pp. 99-151, Berlin: Springer-Verlag, 1993.

[22] E. W. Barankin, "Locally best unbiased estimates," The Annals of Mathematical Statistics, vol. 20, pp. 477-501, Dec. 1949.

[23] R. J. McAulay and L. P. Seidman, "A useful form of the Barankin lower bound and its application to PPM threshold analysis," IEEE Transactions on Information Theory, vol. 15, pp. 273-279, Mar. 1969.

[24] I. Reuven and H. Messer, "The use of the Barankin bound for determining the threshold SNR in estimating the bearing of a source in the presence of another," in Proc. of IEEE International Conference on Acoustics, Speech, and Signal Processing (ICASSP), vol. 3, (Detroit, MI, USA), pp. 1645-1648, May 1995.

[25] R. J. McAulay and E. M. Hofstetter, "Barankin bounds on parameter estimation," IEEE Transactions on Information Theory, vol. 17, pp. 669-676, Nov. 1971.

[26] K. Todros and J. Tabrikian, "General classes of performance lower bounds for parameter estimation - part I: non-Bayesian bounds for unbiased estimators," IEEE Transactions on Information Theory, vol. 56, pp. 50455063, Oct. 2010. 